\title{
Algorithms for Analyzing and Forecasting in a Pharmaceutical Company
}

\author{
Lucelia DEDU \\ Database Master - Business Support \\ Faculty of Economic Cybernetics, Statistics and Informatics \\ Bucharest University of Economic Studies, Romania \\ luceliadedu@yahoo.com
}

This paper presents some of the utilities of using SAS code for analyze and forecast, in a pharmaceutical company. First, data must be cleaned, in order to obtain representative results. Once this stage done, the input can be evaluated and models can be created in SAS, to extract the most valuable information from the initial data. To sustain management decisions, SAS code allows creating different types of reports and has the capability to recode the initial variables into new ones, but keeping the most part of the information contained, through the technique called "principal components analysis". Another advantage of SAS code is its capability of classifying the initial variables into compact class. Based on scorecards and WOE (Weight of Evidence), notions used in banking analyses, it has been created a model which evaluates the suppliers of the company and ranks them, in order to find out what contracts must be continued and which contracts must be closed.

Keywords: Reports, Principal Components Analysis, Clusters, Scorecard, Weight of Evidence

1

\section{Introduction}

Data analysis involves collecting, analyzing and presenting large amounts of data, in order to extract the most valuable information from the initial data and to discover patterns and trends which are used in decision-making process. This means that based on many information, it is important to know how to extract the essence from the available data and how to use it in order to improve the future activity and the results obtained.

Data analysis is a process that involves statistical algorithms or logical techniques to describe and evaluate data. Many of the analyses performed become an iterative process where data is systematically collected and analyzed simultaneously, especially because patterns are discovered through the entire data collection 0 .

Subjecting data to statistical operations can relieve the relationship among variables and the level of trust the answers are getting.

Data analysis is used to process and filter the information contained in the data studied, in order to capture the essence or extract the information contained. Another purpose is to highlight this information in an essentially form, more intelligible, suggestive, simplified and synthesizers. Achieving this goal involves the development of a sequence of transformations performed on the raw data and involves the use of specific methods and techniques. These changes aim to maximize the relevance and interpretability of data and requires, among other things, to eliminate redundant or meaningless information and generality. From this point of view, data analysis appears to be a specific transformation process that takes as input the raw data and as output the information synthesized 0 .

Began in the late 60 's, for analyzing the results of the experiments in agriculture, SAS (Statistical Analysis System) became a powerful programming language, providing data mining tools for Web analysis and development, for a wide range of industries. Nowadays, SAS solutions are used in more than 130 countries, covering areas such as Analytics, Business Intelligence, Customer Intelligence, Data Integration \& ETL, Fraud Prevention \& Detection, Risk Management, Supply Chain Management, Cloud etc. 0.

SAS can import different types of data and creates outputs in diverse formats, being a flexible programming language, which provides statistical, descriptive, inferential 
and predictive analysis.

The base products of SAS are: BASE SAS (data management), SAS / STAT (statistical analysis), SAS/ GRAPH (graphics), SAS/OR (optimization and simulation), SAS/ ETS (time series analysis), SAS/ QC (quality control) and many others.

In SAS, statements are used to write a program, based on instructions, executed in order. SAS statements are grouped into "blocks", referred to as "steps". In a SAS program, we can have data steps or procedure steps. A data step reads and modifies data for analysis, creating a SAS data set, while a procedure step performs a specific utility action on a data set and produces results or print reports. A data set read from an external source and can include DO loops, IF-THEN-ELSE logic and an assortment of numeric and character functions, concatenation and match-merge.

According to 0 , SAS procedures do everything from simple sorting and printing to analysis of variance and 3D graphics.

SAS is known for its flexibility - there aren't many rules about how to format a SAS program: statements can be in uppercase or lowercase, they can continue on the next line (with the condition that they don't split words in two) and they can start in any column. Almost all the statements begin with a keyword which identifies the type of the statement - an exception is the assignment statement which begins with a variable name. But the most important rule remains not to omit the semicolon at the end of the statement 0 .

These statements specify how data are processed and analyzed, present the operations performed on data or detail the instructions about the analysis.

\section{Accessing and Cleaning Data}

In SAS, data can be accessed in 3 different ways:

- Using INFILE declaration;

- Reading data directly from SAS code (for small data sets);

- Using IMPORT procedure.

Table 1. Structure of initial data set

\begin{tabular}{|c|c|c|c|c|c|c|}
\hline Variable & Product & Category & Type & Unitary_price & Range_series & Stock \\
\hline $\begin{array}{c}\text { Variable } \\
\text { type }\end{array}$ & Char(50) & Char(8) & Char(10) & Float(5,2) & Integer(6) & Integer(10) \\
\hline \hline Variable & Sales & Production & Term & Substitutes & Form & Active_ingredients \\
\hline $\begin{array}{c}\text { Variable } \\
\text { type }\end{array}$ & Integer(10) & Integer(10) & Integer(3) & Integer(2) & Char(6) & Integer(2) \\
\hline
\end{tabular}

"Syrup", "Solution" or "Spray".

\begin{tabular}{c|c|c|c|c}
\hline Product & Category & Type & Unitary_price & Range_serie \\
\hline Char(50) & Char(8) & Char(10) & Float(5,2) & Integer(6) \\
& & & & \\
\hline \hline Sales & Production & Term & Substitutes & Form \\
\hline nteger(10) & Integer(10) & Integer(3) & Integer(2) & Char(6) \\
\hline
\end{tabular}

Table 1 presents the variables analyzed. Their description is presented below: Category refers to the status of the pharmaceutical product. This variable can have the values "OTC" - without a prescription, "Rx" - by prescription, "SN" - a dietary supplement, "Cosmetic" or "Biocides".

Type describes the formulation of the product and can have the values "Tablets", "Capsule",
"Initary prtieck Range_series,

Productionterefer) to the production.

Active ingredients number of months of validity gurdegrtiteed for each product.

Substitutes contain the number of products substitued (have the same properties and the same curative effect) for each product.

Form refers to the physical nature of each pharmaceutical product. This variable can have the values "liquid" or solid ".

Active_ingredients contains the number of active ingredients in each product, which influences the shelf life of the products.

Data cleaning is one of the most important steps in processing data, by verifying that the data are correct or at least compliant with a 
certain set of rules. Data cleaning is concerned with detecting and removing errors and incompatibilities to improve data quality. Data quality problems are present in the individual data collections such as files and databases, caused by misspellings during data entry, lack of information or other types of errors.

In order to verify the accuracy of data, it has been created formats for the analyzed variables:

/* Creating formats $* /$

proc format;

value \$Cat_f 'OTC','RX','SN','Cosmetic','Biocide'= 'Valid'

value \$Tip_f

' = 'Gap'

other = 'INVALID',

'Tablets','Capsules','Syrup','Liquid','Spray','Powder' = 'Valid'

' = 'Gap'

other = 'INVALID';

value \$Price_f '0.01'-'999.99' = 'Valid'

' ' = 'Gap'

other = 'INVALID';

value \$Range_f '1'-'99999' = 'Valid'

' $=$ 'Gap';

value \$Stock_f '0'-'999999' = 'Valid'

' $=$ 'Gap'

other = 'INVALID';

value \$Sales_f '0'-'999999' = 'Valid'

' ' = 'Gap'

other = 'INVALID';

value \$Prod_f '0'-'999999' = 'Valid'

' ' = 'Gap'

other = 'INVALID';

value \$Term_f '1'-'99' = 'Valid'

' = 'Gap'

other = 'INVALID';

value \$Subst_f '0'-'50' = 'Valid'

' ' = 'Gap';

value \$Form_f 'liquid','solid' = 'Valid'

' $=$ 'Gap'

other = 'INVALID';

value \$active_f '1'-'99' = 'Valid'

' ' = 'Gap'

other = 'INVALID';

run;

To eliminate records with duplicate values, we can use the code below:

proc sort data=formats.products out=temp;

by Product;

run;

data formats.duplicate formats.nonduplicate;

set temp;

by Product;

if first.Product and last.Product then output

formats.nonduplicate; else output formats.duplicate;

run;

title "Duplicate values: ";

proc print data=formats.duplicate;

id Product;

run;

title "Unique values: ",

proc print data=formats.nonduplicate;

id Product;

run;

\section{Reports}

Once the stage of accessing and cleaning data done, data can be summarized, analyzed and evaluated. Before transform data, it can be created reports to show the initial state. Regarding that data are unique, without missing values and with no incorrect format, the results obtained will be representatives.

Reports are tools for management, which influence the decision making process. SAS offers the possibility of creating such reports, parameterize and customize them, as well as save the resulted reports in PDF or HTML.

The database analyzed belongs to a pharmaceutical company, which means that the information analyzed refer to clients, manufacture, products and suppliers.

For example, we can create a report based on information about products - unit price, category, type, unit stock, unit sales etc.

To create a report about products and the situation of units, considering the category and the type for each product, we can use the code below:

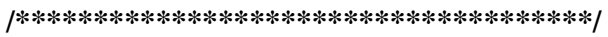

/** REPORT - CATEGORY and TYPE ***/

/******************************************)/

ods pdf

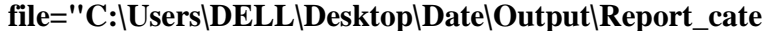

gory_type.pdf" style=sigla;

proc report data=formats.products nowd headline

headskip;

column category type product unitary_price sales stock production;

define category/ group 'Category';

define type / group 'Type';

define product / 'Product';

define unitary_price/group 'Price';

define sales / analysis sum;

define stock / analysis sum;

define production / analysis sum;

break after category / color=red summarize suppress

skip;

break after type / color = blue summarize suppress skip; 
compute after;

line 'Total sales:' sales.sum ' lei (for 2013) ';

line 'Total stock:' stock.sum ' units (for 2013) ';

line 'Total production :' production.sum ' units (for

2013) ';

endcomp;

compute sales;

endcomp;

compute stock; endcomp;

compute production;

endcomp;

where sales > 0;

title "Products and total units of each product";

title2 "by category and type";

run;

ods pdf close;

Products and total units of each product

11:10 Thursdxy, August 25, 2014 by category and type

\begin{tabular}{|c|c|c|c|c|c|c|}
\hline Category & Type & Product & Price & Sales & Stock & Production \\
\hline \multirow[t]{6}{*}{ Biocide } & Liģuid & BETAGN sol.ext. $10 \% 500 \mathrm{ml} \mathrm{xl}$ & 20 & 2082 & 2329 & 2500 \\
\hline & & BETAGN sol.ext. $10 \% 30 \mathrm{ml} x 1$ & 6.5 & 13745 & 24485 & 15000 \\
\hline & & & & 15827 & 26814 & 17500 \\
\hline & Tablets & DEZINFECTANT FORTE compr. $x 50$ & 5.79 & 3678 & 25436 & 7000 \\
\hline & & & & 3678 & 25436 & 7000 \\
\hline & & & & 19505 & 52250 & 24500 \\
\hline \multirow[t]{14}{*}{ Cosmetic } & Capsules & CARMOL BABY caps. x10 & 6.3 & 14299 & 32925 & 10000 \\
\hline & & INHALANT BABY caps. x10 & & 19861 & 61503 & 20000 \\
\hline & & & & 34100 & 94428 & 30000 \\
\hline & Liquid & OSSIDENTA Liquid $50 \mathrm{ml} \mathrm{xl}$ & 3.5 & 65400 & 138022 & 80000 \\
\hline & & INHALANT sol.inh $10 \mathrm{ml} \times 1$ & 3.6 & 151580 & 164822 & 160000 \\
\hline & & OTET AROMAT Liquid $x 1$ & 3.7 & 12237 & 85240 & 12500 \\
\hline & & CARMOL BABY solext. $100 \mathrm{ml} \mathbf{x} 1$ & 4.2 & 14771 & 90881 & 25000 \\
\hline & & OSSIDENTA Liquid $250 \mathrm{ml} \times 1$ Cirese.Menta & 5.35 & 19428 & 50746 & 24000 \\
\hline & & OSSIDENTA Liquid 250ml x1 Menta & & 19428 & 25226 & 16000 \\
\hline & & & & 282844 & 554937 & 317500 \\
\hline & Spray & OSSIDENTA spray $30 \mathrm{ml}$ xl Menta & 6.5 & 5732 & 5889 & 7000 \\
\hline & & OSSIDENTA spray $30 \mathrm{ml}$ xl Cirese.Menta & 6.65 & 5732 & 46181 & 7000 \\
\hline & & & & 11464 & 52070 & 14000 \\
\hline & & & & 328408 & 701435 & 361500 \\
\hline
\end{tabular}

Fig. 1. Report about products and the situation of stock, sales and production units, considering the category and the type for each product

Fig. 1 presents the report which lists by category and type, products and unit prices, while achieving total units sold, the total stock and total units produced. For example, in the category "Biocide", there are 2 types of products - liquid and tablets; the liquid products are Betagin sol. ext. $10 \% 500 \mathrm{ml} \times 1$ and Betagin sol. ext. $10 \% 30 \mathrm{ml} \times 1$ and the tablets are Dezinfectant Forte compr.x50. For every product are listed total units sold, the total stock and total units produced.

\section{Scorecard for Ranking Suppliers}

Scorecard is a technique that allows a company to monitor and manage performance, considering some targets.
Measurements are usually financial performance, customer value, internal business processes, the performance of innovation or employee performance 0 .

Suppliers have an important role in the proper functioning of the Biofarm Company. If suppliers do not fulfill their obligations under the contract, increases the risk that Biofarm reduce or even stop their work due to lack of raw materials or packaging.

Scorecard is used in activities related to risk management of non-commitment to a supplier, in order to decide to conclude a new contract or not to continue to work with suppliers for which risk and losses are related. For these reasons, it is useful to 
review suppliers that Biofarm contracts. The analyze made regarded the top 20 suppliers by value of purchases made by the company. The analysis regards 20 vendors for which 9 attributes are known: Acquisition_value, Term (delivery time agreed in contract), Term(achieved) (delivery time realized), Product_category (the category for each product), Orders_history (the status for each order), Company_type (the type of every supplier), Contract (the length of the collaboration between Biofarm and each supplier), No_of_contracts (number of contracts closed by Biofarm with each supplier), Performance (show if a supplier is performing or not).

Fig. 2 presents the formula for WOE (Weight of Evidence), which represents the power of discrimination of each attribute and helps to differentiate the model risk and must be calculated for each variable.

$$
\text { WOE }=\ln \frac{\frac{\# \text { Performing suppliers from category } X \text { for atribut } Y}{\# \text { Performing suppliers }}}{\frac{\# \text { Underperforming suppliers from category } X \text { for atribut } Y}{\# \text { Underperforming suppliers }}}
$$

Fig. 2. Weight Of Evidence formula

Information Value formula, shown in Fig. 3, has the purpose to select variables during analysis and model building. This concept reflects the overall predictive power of the variables considered, so it can be used for comparing the predictive power among competing variables.

$$
\begin{aligned}
I V=\sum\left(\frac{\# \text { Performing suppliers from category } X \text { for atribut } Y}{\text { \# Performing suppliers }}\right. \\
- \\
\quad \frac{\left.\begin{array}{l}
\text { \#nderperforming suppliers from category } X \text { for atribut } Y \\
\text { Underperforming suppliers }
\end{array}\right)}{\frac{\text { \# Performing suppliers from category } X \text { for atribut } Y}{\# \text { Performing suppliers }}} \\
\text { \# Underperforming suppliers } X \text { for atribut } Y
\end{aligned}
$$

Fig. 3. Information Value formula

Regarding the notions explained above Error!

Reference source not found., the following macro has been defined in order to calculate the Weight of Evidence and the Information Value for each attribute 0 :

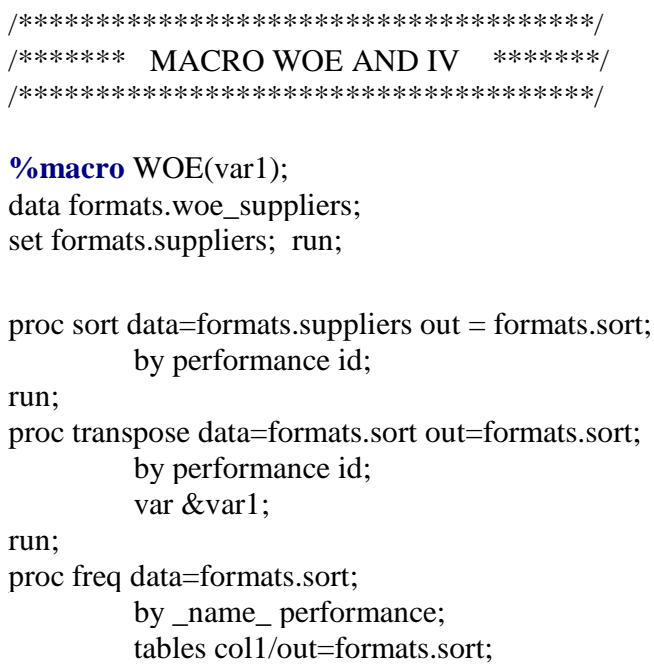

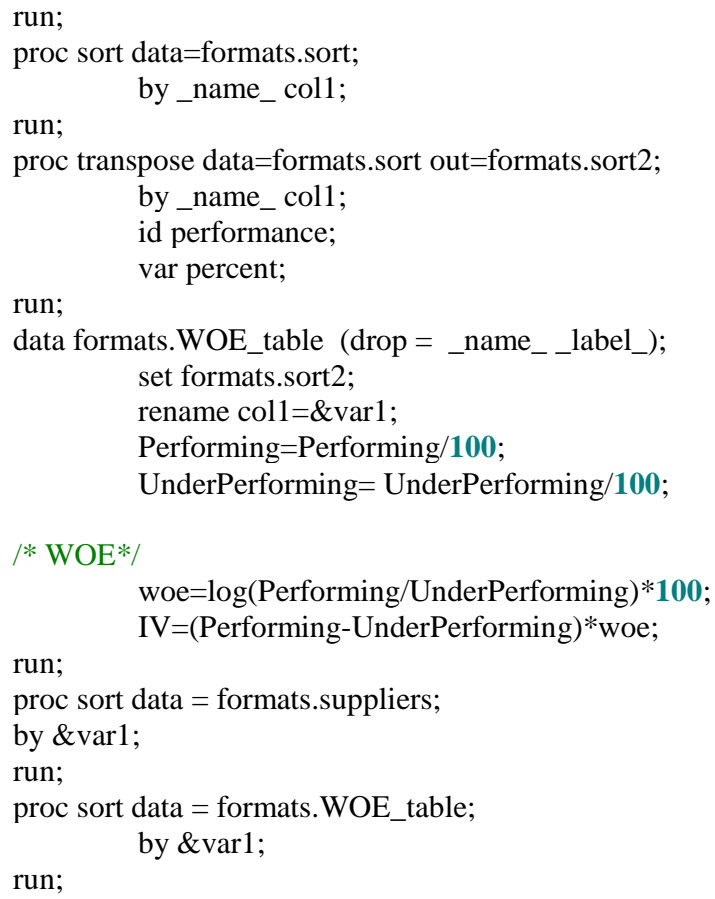


data formats.WOE_SUPPLIERS;

merge formats.suppliers formats.woe_table; by \&var1;

rename woe= \&var1; rename IV=IV_\&var1; drop \&var1;

run;

proc sort data=formats.WOE_SUPPLIERS;

by id; run;

goptions reset $=$ all ctitle $=$ black ftitle $=$ swissb ctext $=$ black htext $=1$ htitle $=2$ ftext $=$ swissb colors $=(\mathrm{cx} 00 \mathrm{cccc} \operatorname{cxcd} 0369 \mathrm{cx} 5 \mathrm{~b} 768 \mathrm{~d}$ cx594f4a cx008080 cxff8f71)

transparency

iback='C:IUsers\DELLIDesktop\bio2.png' imagestyle=fit; title 'WOE -- ' \&var1;

axis1 label=(\&var1);

As it is shown in Fig. 4, the biggest negative value for the Acquisition_value variable is obtained for the "300-600 mil" category, which means that the category of dealers who have obtained orders worth 300-600 thousand, there are more underperforming suppliers than performing. The ratio of nonperforming providers were able to breach of axis2 label= ('WOE');

proc gchart data=formats.WOE_table;

hbar3d \&var1 / sumvar = woe maxis=axis1 raxis=axis2

discrete;

run;

quit;

\% mend WOE;

$\% \boldsymbol{W O E}$ (Acquisition_value);

$\% \boldsymbol{W O E}$ (Term);

\%WOE(Term_achieved);

$\%$ WOE (Product_category)

\%WOE(Orders_history);

\% WOE (Company_type);

$\%$ WOE (Contract);

\%WOE(No_of_contracts);

contract is lower for suppliers regarding Acquisition_value attribute than the whole portfolio.

The graphic shows that the most reliable and profitable suppliers are those who obtained orders worth more than 1 million or less than 300 thousand.

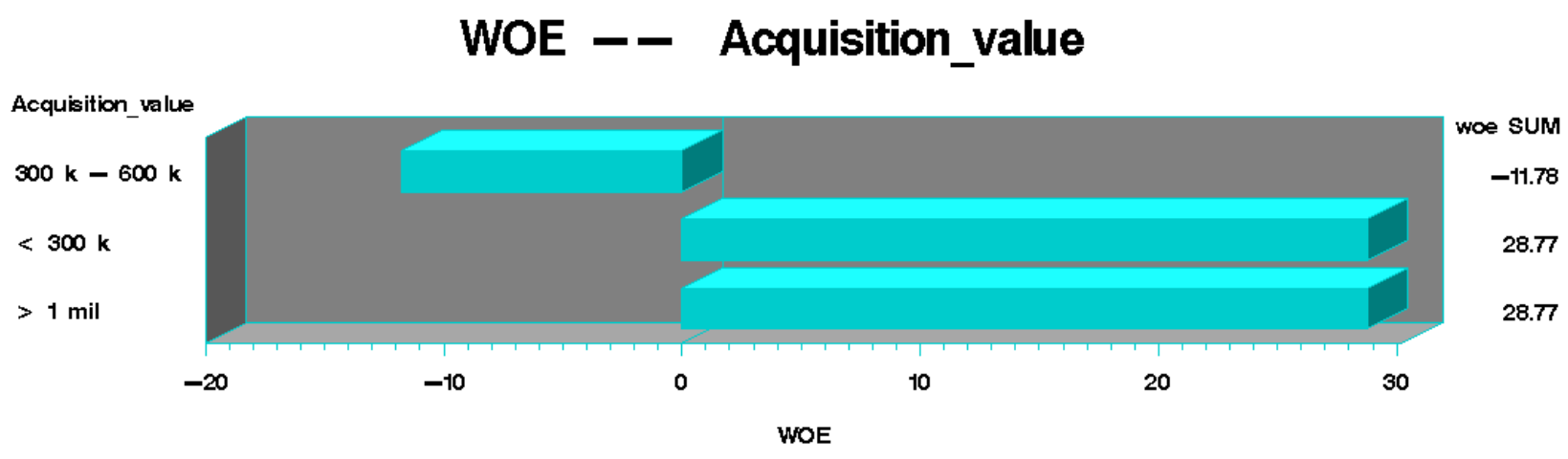

Fig. 4. Weight of Evidence for the variable Acquisition_value

As it is presented in Fig. 5, the biggest negative value for the variable Term is obtained for the "45" category, which means that for the category of providers who should deliver in 45 days, there are more underperforming suppliers than performing ones. The chart above shows that the most profitable are providers who delivered the order within 30 days.

\section{WOE - - Term}

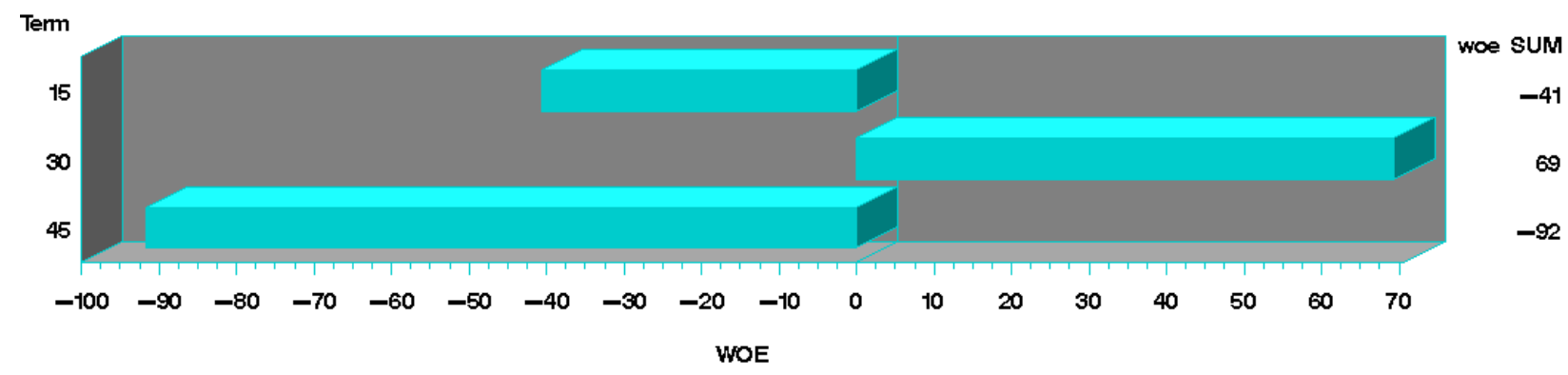

Fig. 5. Weight of Evidence for the variable Term 
According to 0 and 0 , in order to eliminate the missing values from the table created, the code below must be run:

proc stdize data=fz.WOE_FZ reponly missing=0

out=fz.WOE_FZ;

var_numeric_; run;

data fz.WOE_global; set fz.WOE_FZ; drop performant; drop neperformant; IV=IV_Val_Achizitii +

IV_Termen_plata + IV_Termen_realizat +

IV_Cat_Produse + IV_Istoric_plata + IV_Tip_societate + IV_Durata_Contract + IV_Nr_Contracte; run;

\begin{tabular}{|rlr|}
\hline ID & Supplier & \multicolumn{1}{c|}{ IV } \\
\hline 1 & Bostocke Trading LTD & 50.028 \\
2 & Teva Czech Industries s.r.o. & 88.820 \\
3 & XIAMEN FOREVER GREEN SOURCE BIOCHEM & 60.843 \\
4 & Colorcon Limited & 61.496 \\
5 & Indena SAS & 95.638 \\
6 & PHYTOPHARM KLEKA SA & 93.675 \\
7 & Selectchemie AG & 135.388 \\
8 & S \& D Chemicals LTD & 112.066 \\
9 & ICE SPA & 131.113 \\
10 & AIS PRODIMPEX S.R.L. & 104.568 \\
11 & BASF CHEM TRADE GMBH & 85.393 \\
12 & MORGAN THORPE SA & 113.077 \\
13 & Linnea SA & 39.316 \\
14 & DSM Nutritional Products Europe LTD & 37.192 \\
15 & Barentz Romania SRL & 70.032 \\
16 & TIPOGRAFIA POLIROM SRL & 114.251 \\
17 & Kunststoffwerk Kremsmunster GMBH & 83.977 \\
18 & ARGOROM PLASTICS & 42.971 \\
19 & SGD(SAINT GOBAIN) & 99.265 \\
20 & ONGROPACK KFT.BC-ONGROPACK LTD & 126.097 \\
\hline
\end{tabular}

Fig. 6. Information Value, for every supplier

By summing the Information Value for each attribute, as in Fig. 6, Information Value can be calculated globally as running the following code, suggested in 0 :

proc sql;

create table formats.RANK

as select id, supplier, performance, IV from

formats.WOE_global;

run;

proc rank data $=$ formats. RANK out $=$ formats. RANK descending;

var iv;

ranks rank_number;

run;

proc sort data $=$ formats. RANK out $=$ formats. RANK ; by rank_number;

run;

data formats.RANK2;

set formats.RANK;

rename rank_number $=$ rank;

run;

proc template;

define style sigla; parent=styles.printer;

style header from header /

background=_undef_;

style body from document /

background=_undef_

backgroundimage="C:IUsers\DELLIDesktoplsigla.jpg";

end;

run;

ods pdf

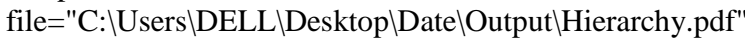
style=sigla;

title 'Suppliers hierarchy';

title2 ' - using Information Value criteria -';

proc print data $=$ formats. RANK;

run;

goptions reset $=$ all $\mathrm{ctitle}=\mathrm{black}$ ftitle $=\mathrm{swissb}$ ctext=black htext $=\mathbf{1}$ htitle $=\mathbf{2}$ ftext $=$ swissb colors $=(\mathrm{cx} 00 \mathrm{cccc} \operatorname{cxcd} 0369 \mathrm{cx} 5 \mathrm{~b} 768 \mathrm{~d}$ cx594f4a cx008080 cxff8f71)

transparency

iback='C: $\backslash$ Users $\backslash$ DELL $\backslash$ Desktoplbio2.png' imagestyle=fit; title 'Suppliers hierarchy';

axis1 label=('Rank');

axis2 label= ('Information Value');

proc gchart data $=$ formats. RANK;

hbar3d rank_number supplier / sumvar = iv maxis=axis 1 raxis=axis2 discrete;

run;

quit;

ods pdf close;

Based on nine attributes recorded for the 20 suppliers, the Weight of Evidence has been calculated for each attribute and the information has been reflected by each feature separately. Considering the products, their category, type, unitary_price, range_series, stock, sales, production, term, substitutes, form and active_ingredients, the Information Value compares the predictive power across those 9 attributes and the Weight of Evidence reflects how behaves every variable considered.

As shown in Fig. 7, the information aggregated globally has revealed a hierarchy of suppliers, after descending the Information Value field. 


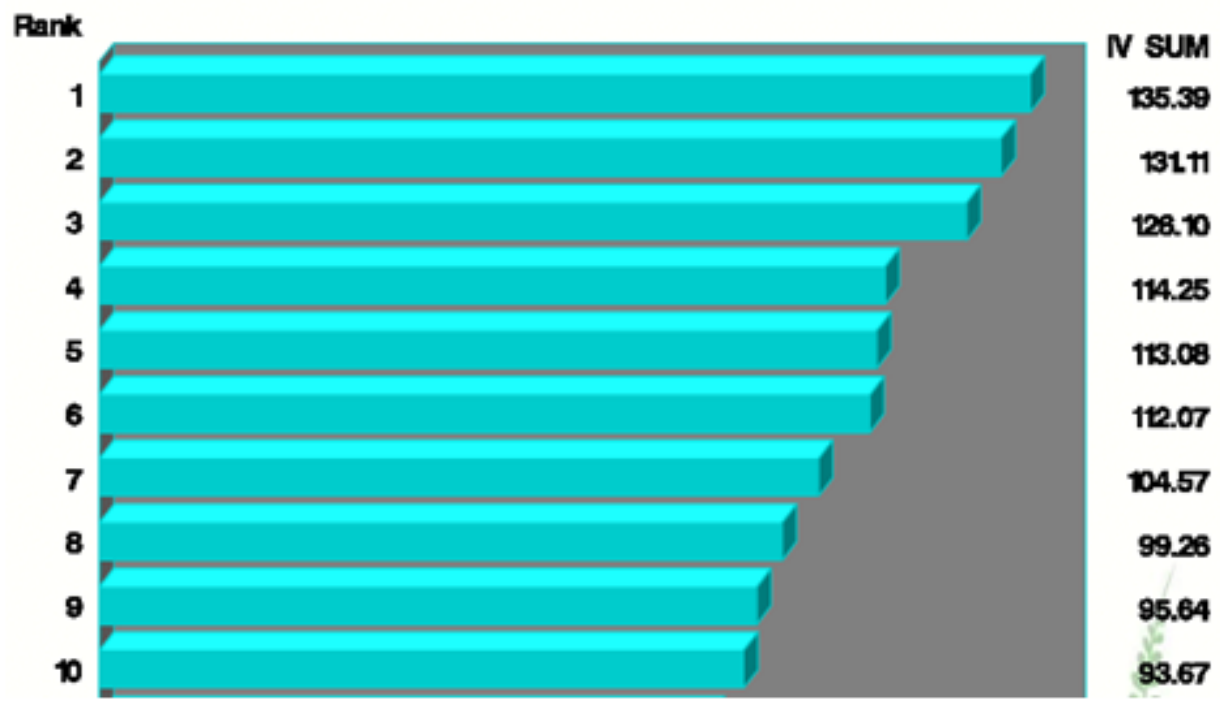

Fig. 7. Suppliers hierarchy, by Information Value

The report in Fig. 8 highlight the suppliers The report presented above facilitates with whom Biofarm need to maintain their management decisions, providing a clear collaboration, due to good results obtained in situation of the suppliers, considering the the past. variables analyzed.

Suppliers hierarchy - using Information Value criteria -

\begin{tabular}{|c|c|c|c|c|}
\hline Obs & ID & Supplier & IV & rank_number \\
\hline 1 & 7 & Selectehemie AG & 135.388 & 1 \\
\hline 2 & 9 & ICE SpA & 131.113 & 2 \\
\hline 3 & 20 & ONGROPACK KFT BC-ONGROPACK LTD & 126.097 & 3 \\
\hline 4 & 16 & TIPOGRAFLA POLIROM SRL. & 114.251 & 4 \\
\hline 5 & 12 & MORGAN THORPE SA & 113.077 & 5 \\
\hline 6 & 8 & S \& D Chemicals LTD & 112.066 & 6 \\
\hline 7 & 10 & AIS PRODLMPEX SRL. & 104.568 & 7 \\
\hline 8 & 19 & SGD(SANT GOBAN) & 99.265 & 8 \\
\hline 9 & 5 & Indena SAS & 95.638 & 9 \\
\hline 10 & 6 & PHYTOPHARM KLEKA SA & 93.675 & 10 \\
\hline 11 & 2 & Teva Czech Industries s. . o. & 88.820 & 11 \\
\hline 12 & 11 & BASF CHEM TRADE GMBH & 85.393 & 12 \\
\hline 13 & 17 & Kunststoffwerk Kremsmunster GMBH & 83.977 & 13 \\
\hline 14 & 15 & Barentz Romania SRL. & 70.032 & 14 \\
\hline 15 & 4 & Colorcon Limited & 61.496 & 15 \\
\hline 16 & 3 & XIAMEN FOREVER GREEN SOURCE BIOCHEM & 60.843 & 16 \\
\hline 17 & 1 & Bostocke Trading LTD & 50.028 & 17 \\
\hline 18 & 18 & ARGOROM PLASTICS & 42.971 & 18 \\
\hline 19 & 13 & Linnea SA & 39.316 & 19 \\
\hline 20 & 14 & DSM Nutritional Products Europe LTD & 37.192 & 20 \\
\hline
\end{tabular}

Fig. 8. Suppliers hierarchy, by Information Value

As stated in the report above, top 5 suppliers for Biofarm are Selectchemie AG, ICE SpA, 
Ongropack Kft BC, Tipografia Polirom SRL and Morgan Thorpe SA. Considering the results obtained, Biofarm will continue to collaborate with those companies and can close the contracts with the suppliers which didn't fulfill their responsibilities, such as Xiamen Forever, Bostocke, Argorom Plastics, Linnea SA or DSM Nutritional Products Europe LTD.

\section{Conclusions}

In the beginning of this article, SAS has been presented as an important data analysis software tool, dedicated to highlight the power and importance of using such programs for statistical analysis of the data in a company.

Following, it has been shown the importance of data cleaning and validation; this phase cannot miss because the analyze need accurate data in order to obtain representative results. For that, it has been created data validation formats and the records which didn't comply have been removed.

Once the stage of accessing and cleaning data done, data can be summarized, analyzed and evaluated. Before transform data, it can be created reports to show the initial state. Regarding that data are unique, without missing values and with no incorrect format, the results obtained will be representatives.

Reports defined in SAS allow visualization of key information about products and are useful tools for sustaining management decisions. SAS offers the possibility of creating such reports, parameterize and customize them, as well as save the resulted reports in PDF or HTML.

Scorecard created for the company's suppliers has the purpose to sustain management decisions. The hierarchy resulted takes into account the performance indicators obtained in the last year, and historical data, such as length collaboration between the company and suppliers, the number of contracts and the degree of respect the contract by each supplier.

\section{References}

[1] F. D. Delwiche and S. J. Slaughter, "The Little SAS Book: A Primer: A Programming Approach", 2012

[2] G. Ruxanda, "Data Mining", Bucharest, 2010

[3] Information about SAS products, available:http://www.sas.com/en_us/com pany-information.html\#stats

[4] G. Der and B. S. Everitt, "A Handbook of Statistical Analyses using SAS", 2002

[5] M. Evans, "SAS Manual For Introduction to the Practice of Statistics", 2010

[6] Information about variable reduction in SAS, using WOE (Weight of Evidence) and IV (Information Value): http://support.sas.com/resources/papers/p roceedings 13/095-2013.pdf

[7] Information about WOE (Weight of Evidence) and IV (Information Value): http://documentation.statsoft.com/STATI STICAHelp.aspx?path=WeightofEvidenc e/WeightofEvidenceWoEIntroductoryOv erview

[8] F. E. Pratter, "Web Development with SAS® by Example", 2006

[9] J. Shostak, "SAS $®$ Programming in the Pharmaceutical Industry", 2005

[10] R. Cody, "Learning SAS $®$ by Example A Programmer's Guide", 2007

[11] SAS Institute Inc. Step-by-Step Programming with Base SAS $®$ Software, 2001 


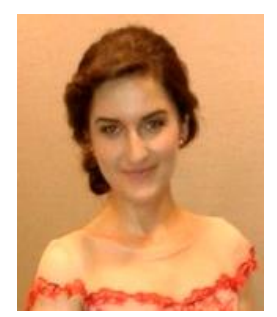

Lucelia DEDU has graduated the Faculty of Economic Cybernetics, Statistics and Informatics from the Bucharest Academy of Economic Studies in 2012 and she has graduated in 2014 the courses of "Databases for Business Support" master program organized by the Academy of Economic Studies of Bucharest. During her second year at the master program, she followed the course Credit Risk Management, held by BCR, where she discovered new features of using SAS code. Her career is centered on payroll domain, database's queries, ETL Tools for data warehouses and COBOL development. Her areas of interest are: Data Warehouses, Data Modeling, Risk Management, Databases and SAS development. 